Supporting Information

\title{
An autonomous nonenzymatic concatenated DNA circuit for amplified imaging of intracellular ATP
}

Yangjie Zhou, Lei Yang, Jie Wei, Kang Ma, Xue Gong, Jinhua Shang, Shanshan Yu and Fuan Wang *

Key Laboratory of Analytical Chemistry for Biology and Medicine (Ministry of Education), College of Chemistry and Molecular Sciences, Wuhan University, Wuhan, P. R. China

* To whom correspondence should be addressed. E-mail: fuanwang@whu.edu.cn. 


\section{Supporting Information}

\section{Table of Contents}

Figure S1. Construction of upstream HCR-1 circuit.

Figure S2. Construction of downstream HCR-2 circuit...............................................................

Figure S3. Validation of ATP-triggered Ca-HCR aptasensor...........................................................

Figure S4. Demonstration of Ca-HCR amplification..................................................................S6

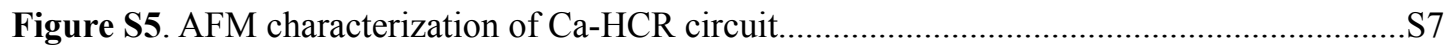

Figure S6. Optimization of ATP-motivated Ca-HCR aptasensor..................................................S8

Figure S7. Optimization of incubation time for Ca-HCR imaging platform.................................S9

Figure S8. Demonstration of Ca-HCR-mediated intracellular ATP imaging.................................S10

Figure S9. Exploration of intracellular ATP fluctuation.............................................................. 11

Equation S1. Reaction simulation of the ATP-mediated Ca-HCR sensor......................................S12

Table S1. Reaction constants as acquired from the theoretical simulations.....................................S15

Table S2. DNA sequences of the Ca-HCR-amplified ATP aptasensor.............................................S16

Table S3. Summary of the different fluorescent ATP aptasensors................................................. 17

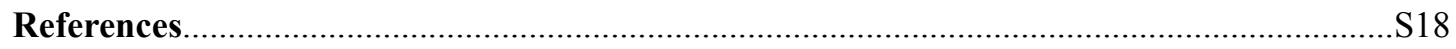




\section{Construction of upstream HCR-1 circuit}

The ATP-motivated upstream HCR-1 reaction involves two transducing stages: initial identification and subsequent hybridization. The cross-hairpin hybridization is activated upon a successful execution of ATP identification, as schematically depicted in Figure S1. The AptInh strand releases from the AptC/AptInh hybrid after the recognition of ATP with its aptamer, which can expose initiator strand for initial hybridization chain reaction (HCR-1). There are two hairpins $\mathbf{H}_{\mathbf{1}}$ and $\mathbf{H}_{\mathbf{2}}$ in HCR-1 system. $\mathbf{H}_{\mathbf{1}}$ includes the sequence $\mathrm{b}^{*}$ (pink) that is complementary to initiator I (b), while $\mathbf{H}_{2}$ includes domain $\mathrm{d}^{*}$ (green) that is complementary to sequence d (green) of $\mathbf{H}_{\mathbf{1}}$. Hairpins $\mathbf{H}_{\mathbf{1}}$ and $\mathbf{H}_{\mathbf{2}}$ are further elongated with domains $\mathrm{f}$ and e at its 5'- and 3'-ends, respectively. Then the exposed initiator opens domain $\mathrm{b}^{*}$ of hairpin $\mathbf{H}_{\mathbf{1}}$ to generate $\mathbf{I}-\mathbf{H}_{\mathbf{1}}$ hybrid via a toeholdmediated strand displacement mechanism, resulting in the newly exposed sticky domain d of $\mathbf{H}_{\mathbf{1}}$ to open $\mathbf{H}_{2}$. Then the formation of an intermediate structure $\mathbf{I}-\mathbf{H}_{\mathbf{1}}-\mathbf{H}_{\mathbf{2}}$ brings the separated segments $\mathbf{f}$ and e into close proximity, leading to the assembly of dsDNA nanowires analogous with numerous repeated structures $\mathbf{T}$ (domain f-e). Thus the ATP-triggered upstream HCR-1 reaction successively produces the reconstituted colocalized structure $\mathbf{T}$ which then triggers downstream HCR-2 reaction.

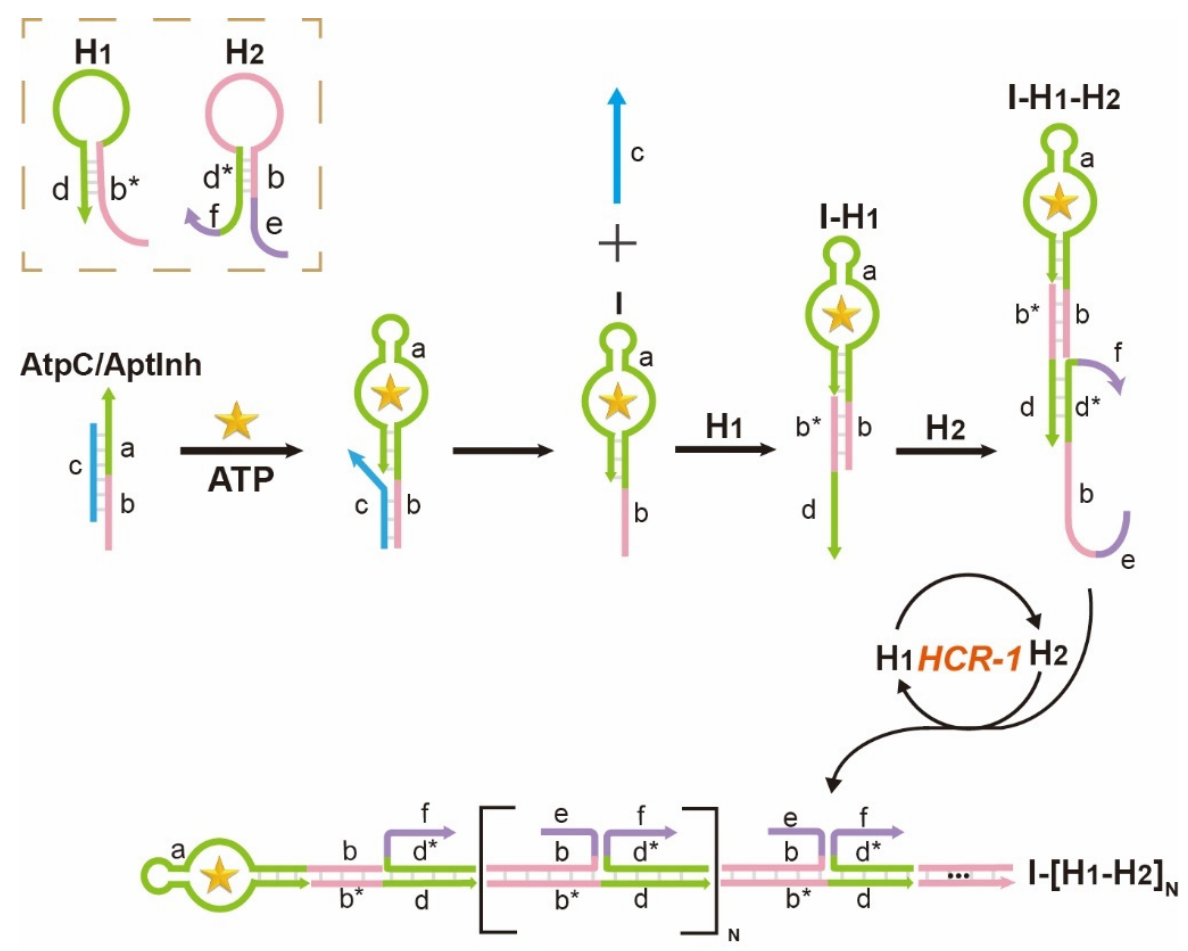

Figure S1. The schematic diagram of the ATP-triggered upstream HCR-1 circuit. 


\section{Supporting Information}

\section{Construction of downstream HCR-2 circuit}

As seen in Figure S2, the downstream HCR-2 reaction is composed of four DNA hairpins: $\mathbf{H}_{\mathbf{3}}$, $\mathbf{H}_{4}, \mathbf{H}_{5}$ and $\mathbf{H}_{6}$. Here TAMRA is labeled at $3^{\prime}$-end of $\mathbf{H}_{3}$ as a fluorescence acceptor, while FAM is labeled at 5'-end of $\mathbf{H}_{5}$ as a fluorescence donor. Upon the formation of the tandem repeated structure T from upstream HCR-1, the reconstituted colocalized structure T (domain e-f) opens TAMRAlabeled $\mathbf{H}_{3}$ for forming an intermediate $\mathbf{T}-\mathbf{H}_{3}$ structure via a toehold-mediated strand displacement. Then the $\mathbf{T}-\mathbf{H}_{3}-\mathbf{H}_{4}$ structure is generated after the exposed domain g-e of $\mathbf{H}_{3}$ hybridizes with domain $\mathrm{g}^{*}$-e* of $\mathbf{H}_{\mathbf{4}}$. Afterwards, the released domain e-h of $\mathbf{H}_{\mathbf{4}}$ unfolds FAM-labeled $\mathbf{H}_{5}$ with a sticky end of $\mathrm{e}^{*}$-h*, which facilitates the formation of an intermediate hybrid $\mathbf{T}-\mathbf{H}_{3}-\mathbf{H}_{4}-\mathbf{H}_{5}$. This can bring FAM and TAMRA into close proximity and allows for the generation of an efficient Förster resonance energy transfer (FRET) process. The exposed domain i-e of $\mathbf{H}_{5}$ opens $\mathbf{H}_{\mathbf{6}}$ to construct an intermediate T- $\mathbf{H}_{3}-\mathbf{H}_{4}-\mathbf{H}_{5}-\mathbf{H}_{6}$ hybrid, which exposes a single-stranded domain e-f of $\mathbf{H}_{6}$ with an analog sequence of the colocalized structure $\mathbf{T}$. Thus the exposed sequence of $\mathbf{H}_{\mathbf{6}}$ leads to HCR-2-involved multiple assembly of $\mathbf{H}_{3}, \mathbf{H}_{4}, \mathbf{H}_{5}$ and $\mathbf{H}_{6}$ into long dsDNA copolymers consisting of a large amount of adjacent FAM/TAMRA pairs with tremendously amplified FRET signal.

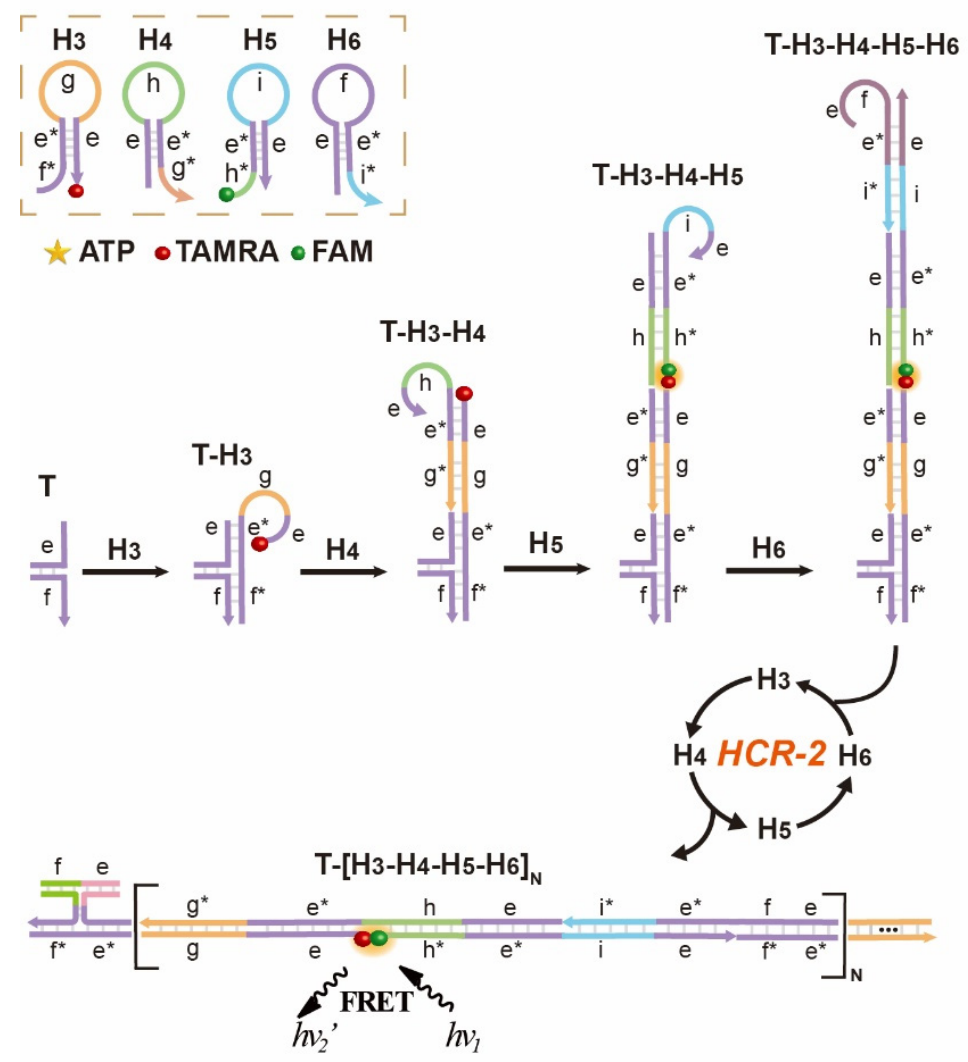

Figure S2. The schematic diagram of the downstream HCR-2 circuit. 


\section{Supporting Information}

\section{Validation of ATP-triggered Ca-HCR aptasensor}

The ATP-triggered Ca-HCR aptasensor was verified by fluorescence assay. The AptC strand has two parts: the aptamer domain and the Ca-HCR trigger domain. Importantly, the trigger sequence of AptC strand is blocked by the AptInh strand. An obvious signal leakage will reveal if the trigger domain of AptC strand is exposed without blockage. As shown in Figure S3, the fluorescence of the non-blocked aptasensing system decreased with and without ATP (curve a and b) with no ATP-sensing ability. Similarly, without the participation of hairpins $\mathbf{H}_{\mathbf{1}}$ and $\mathbf{H}_{\mathbf{2}}$ (curve d and $\mathrm{f}$ ), the upstream HCR-1 cannot be activated to obtain tandem colocalized triggers (T) for promoting downstream HCR-2 even the AptC strand is effectively blocked by AptC/AptInh hybrid. Thus ATP-triggered HCR-1 generates tandem colocalized triggers (T) for downstream HCR-2 that mediates the assembly of frond-like branched copolymeric dsDNA nanowires. Through HCR-2assembled long DNA concatemer, these FAM and TAMRA fluorophores can be brought into close proximity and produce a remarkable FRET signal (curve $\mathrm{c}$ and e).

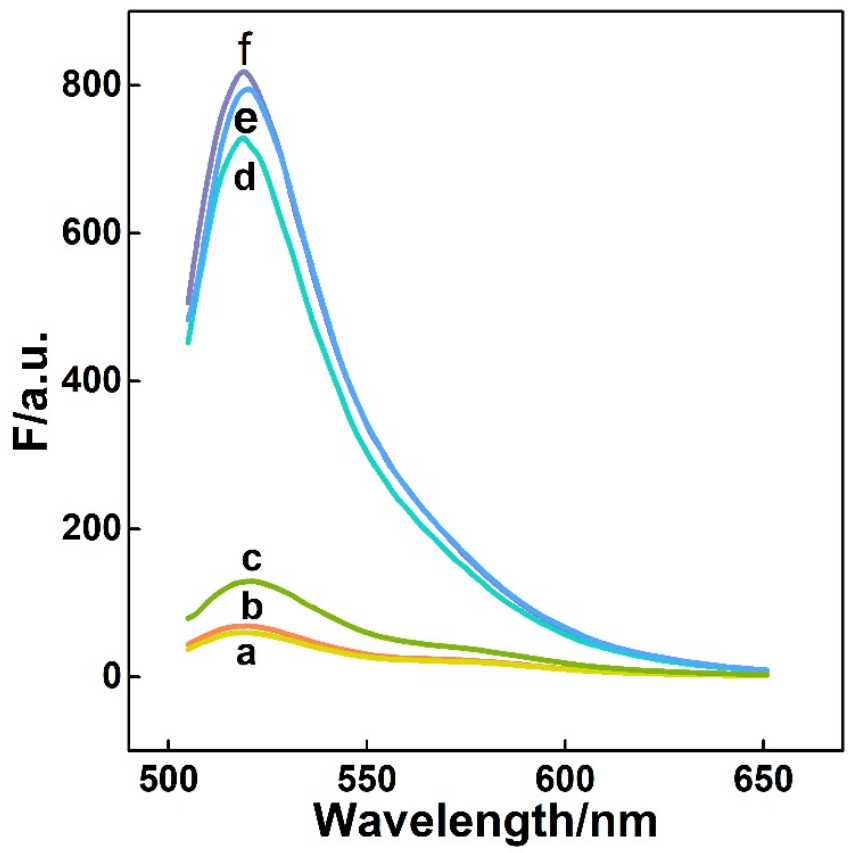

Figure S3. Fluorescence spectra generated by the Ca-HCR sensor with merely AptC strand (a. 500 $\mu \mathrm{M}$ ATP, b. no ATP), AptC/AptInh hybrid without $\mathbf{H}_{\mathbf{1}}$ and $\mathbf{H}_{\mathbf{2}}$ (d. $500 \mu \mathrm{M}$ ATP, f. no ATP), AptC/AptInh hybrid (c. $500 \mu \mathrm{M}$ ATP, e. no ATP). These lines of fluorescence spectra were acquired after these different Ca-HCR mixtures were carried out in reaction buffer (10 mM HEPES, $1 \mathrm{M}$ $\mathrm{NaCl}, 10 \mathrm{mM} \mathrm{MgCl} 2, \mathrm{PH}$ 7.2) for a fixed time-interval of $5 \mathrm{~h}$. 


\section{Supporting Information}

\section{Demonstration of Ca-HCR amplification}

The significant signal gain of our proposed Ca-HCR-amplified sensing platform is dominated by the sequential and successive cross-opening of the corresponding hairpin mixtures. Both of HCR1 and HCR-2 reactions are indispensable for executing the efficient $\mathrm{Ca}-\mathrm{HCR}$ system. Thus the $\mathrm{Ca}-$ HCR amplifier cannot be activated once the non-fluorescent hairpin monomer $\left(\mathbf{H}_{1}, \mathbf{H}_{2}, \mathbf{H}_{4}\right.$, or $\left.\mathbf{H}_{6}\right)$ is removed from the Ca-HCR system. As seen in Figures S4, no apparent fluorescence changes were obtained for ATP-motivated Ca-HCR aptasensor by subtracting $\mathbf{H}_{\mathbf{1}}$ or $\mathbf{H}_{\mathbf{2}}$ from upstream HCR1 reaction (data a and $b$ ) or by subtracting hairpin $\mathbf{H}_{4}$ from downstream HCR-2 reaction (data c). However, a minimal growth of fluorescence was observed for the ATP-triggered Ca-HCR sensor without $\mathbf{H}_{6}$ (data d). It is reasonable since the $\mathbf{H}_{6}$-expelled $\mathrm{Ca}$-HCR circuit corresponds to a conventional HCR system through the stoichiometric FRET readout of HCR-1 scheme. This singlelayered HCR is encoded with a much lowered signal amplification efficacy as compared with the Ca-HCR amplifier (data e). Evidently, the Ca-HCR aptasensor can only be activated with both upstream HCR-1- and downstream HCR-2-involved reactants to facilitate the amplified detection of trace amount of target.
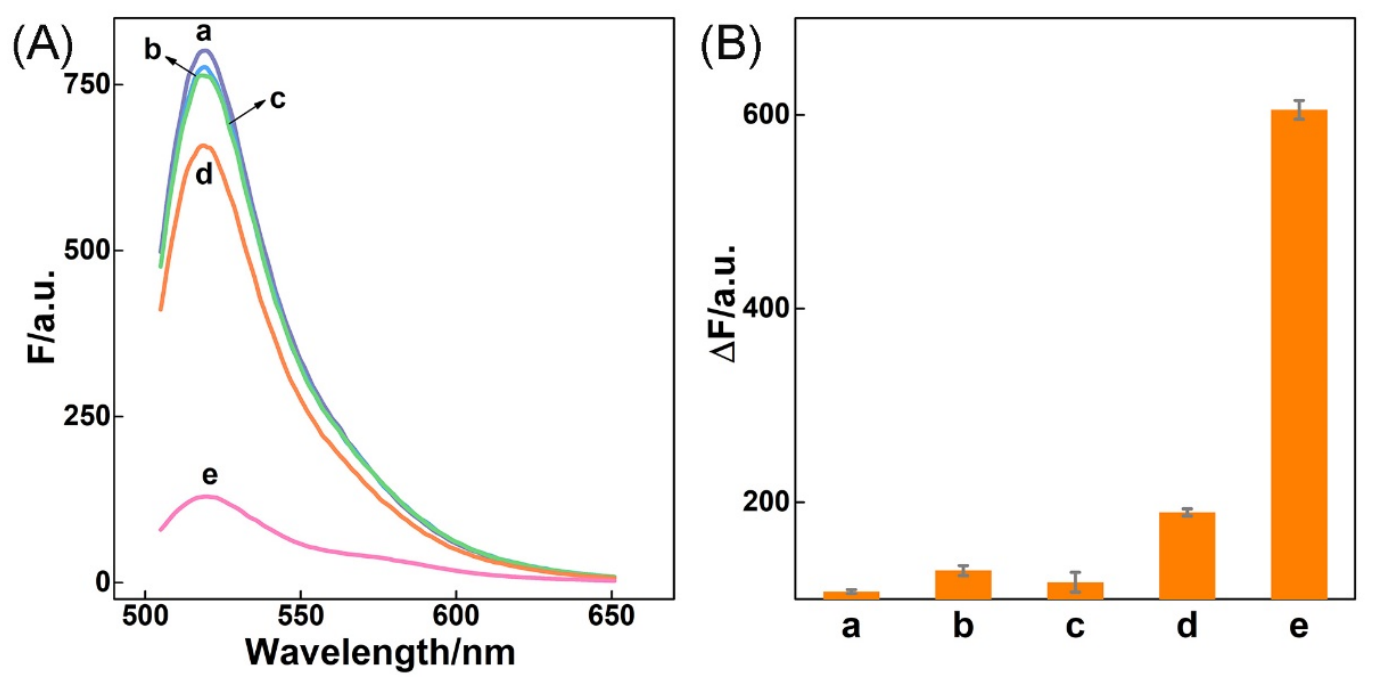

Figure S4. (A) Fluorescence spectra generated by the $500 \mu \mathrm{M}$ ATP-targeting Ca-HCR aptasensor system without hairpin $\mathbf{H}_{1}$ (a), $\mathbf{H}_{2}$ (b), $\mathbf{H}_{\mathbf{4}}$ (c), or $\mathbf{H}_{\mathbf{6}}$ (d). The intact HCR system was introduced as a control (e). (B) The corresponding statistical analysis of fluorescence spectra $(\lambda=520 \mathrm{~nm})$ of these different revised $\mathrm{Ca}-\mathrm{HCR}$ systems. Error bars were derived from $\mathrm{n}=3$ experiments. 


\section{Supporting Information}

\section{AFM characterization of Ca-HCR circuit}

Atomic force microscopy (AFM) was used to investigate the morphology of Ca-HCRgenerated DNA nanostructures (Figure S5). Many tiny spots of the Ca-HCR hairpin monomers were existed since no product is assembled without the Ca-HCR initiator (Figure S5A). Yet long branched DNA polymeric nanowires were observed for the triggered Ca-HCR circuit (Figure S5B) and the triggered HCR-1 reaction shows a long linear DNA structure (Figure S5C). The height of the DNA nanostructure was measured to be $\sim 1.5 \mathrm{~nm}$, a characteristic height of dsDNA (Figure S5D). It is reasonable for the branched dsDNA nanowires are integrated by $\mathrm{Ca}-\mathrm{HCR}$ circuit that was consisting of HCR-1 and HCR-2 reactions. Clearly, the successful implementation of Ca-HCR circuit was demonstrated with significant high signal amplification capacity.
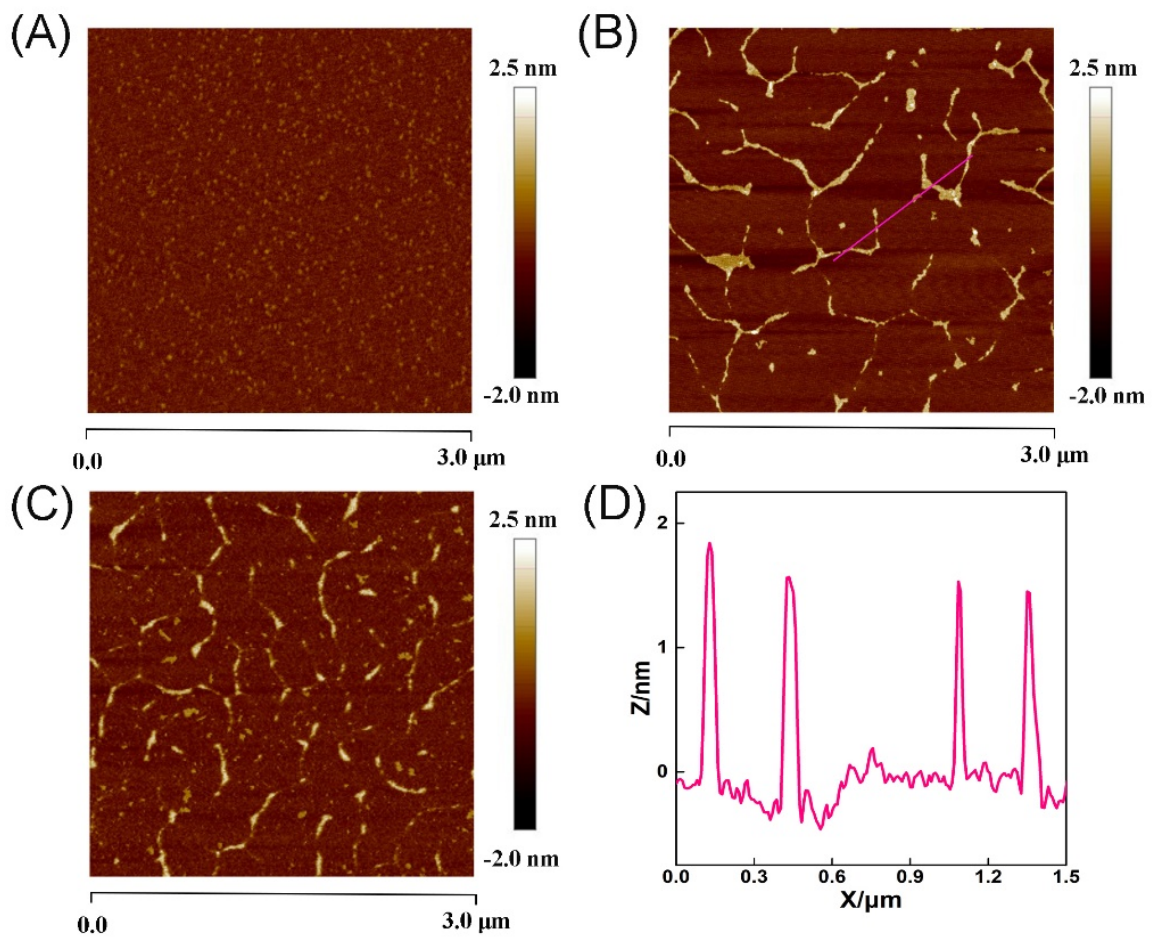

Figure S5. AFM images of non-activated Ca-HCR mixture (A), activated Ca-HCR system (B) and HCR-1-generated product (C). Cross-section analysis of the Ca-HCR-generated branched dsDNA nanowires (D). The Ca-HCR sample was prepared in reaction buffer (10 mM HEPES, $1 \mathrm{M} \mathrm{NaCl}$, $10 \mathrm{mM} \mathrm{MgCl}_{2}, \mathrm{pH}$ 7.2). 


\section{Optimization of ATP-motivated Ca-HCR aptasensor}

The environmental condition plays an important role for achieving an effective ATP-sensing performance. Firstly, the captured ATP is based on a specific interaction between ATP and its aptamer, through which the trigger strand can be exposed from the AptC/AptInh hybrid. As seen in Figure S6A, the effect of AptC concentration was explored on the fluorescence response of our Ca-HCR aptasensor when the AptC/AptInh ratio was kept at 1:2. The optimized ATP fluorescence response $\left(\mathrm{F}_{0}-\mathrm{F}\right)$ was obtained at $80 \mathrm{nM}$ AptC and $160 \mathrm{nM}$ AptInh. The ratio of AptC strand and AptInh strand was then explored with the optimal sensing performance at 1:2 for effective ATP assay (Figure S6B). Moreover, the reaction time also plays a vital role in the ATP assay system. The absolute change of fluorescence intensity $\left(\mathrm{F}_{0}-\mathrm{F}\right)$ increased gradually with prolonged reaction time under $25^{\circ} \mathrm{C}$, and reached to a plateau after $5 \mathrm{~h}$ (Figure S6C). Accordingly, $5 \mathrm{~h}$ was chosen as the optimized reaction time for the following experiments.
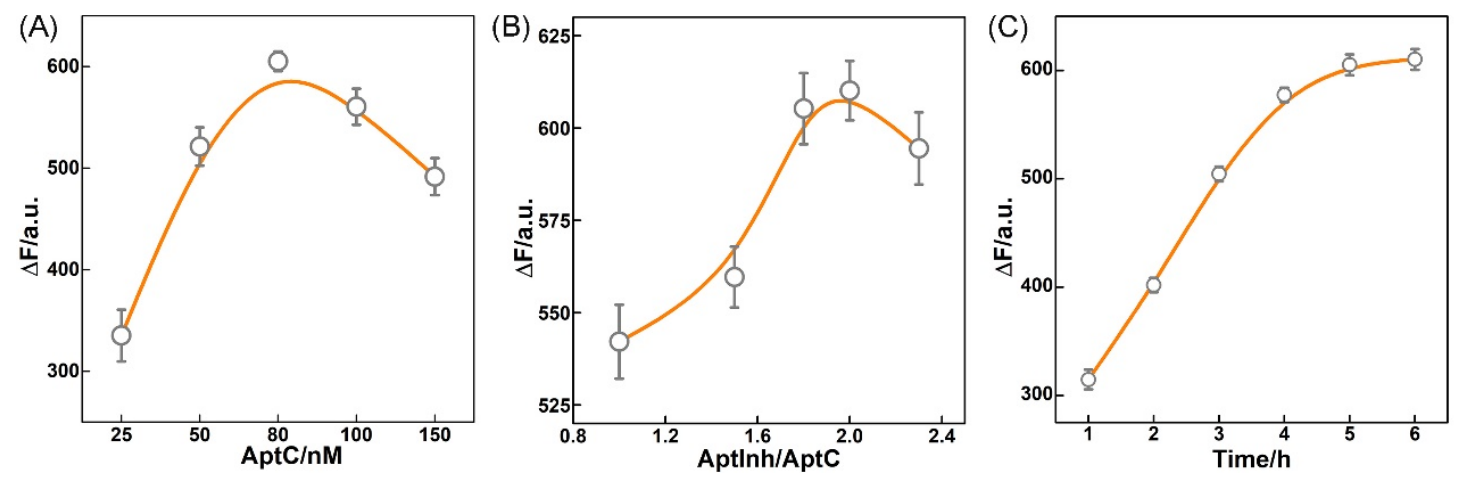

Figure S6. (A) Optimization of the AptC concentration while keeping a constant AptC/AptInh ratio at 1:2. (B) Optimization of the proportion of AptC/AptInh hybrid while keeping a constant AptC concentration at $80 \mathrm{nM}$. (C) Optimization of the incubation reaction as revealed by fluorescence measurements $(\lambda=520 \mathrm{~nm}) .500 \mu \mathrm{M}$ ATP was incubated with the Ca-HCR-based homogeneous ATP sensing platform at $25{ }^{\circ} \mathrm{C}$. The Ca-HCR system consisting of $300 \mathrm{nM} \mathrm{H}_{\mathbf{1}}, 300$ $\mathrm{nM} \mathrm{H}_{2}, 300 \mathrm{nM} \mathrm{H}_{3}, 200 \mathrm{nM} \mathrm{H}_{4}, 200 \mathrm{nM} \mathrm{H}_{5}$ and $200 \mathrm{nM} \mathrm{H}_{6}$ was introduced in reaction buffer for exploration of the optimal reaction condition. Error bars were derived from $\mathrm{n}=3$ experiments. 


\section{Optimization of incubation time for Ca-HCR imaging platform}

To maximize the amplification efficiency of our intracellular ATP imaging system, HeLa cells was incubated with the Ca-HCR aptasensor by lipofectamine 3000 transfection for different timeintervals at $37{ }^{\circ} \mathrm{C}$ to search the most appropriate incubation time. Then these HeLa cells were washed with DPBS three times to remove excess DNA before CLSM observations. As shown in Figure S7, a weak FRET readout $\left(\mathrm{F}_{\mathrm{A}} / \mathrm{F}_{\mathrm{D}}\right)$ was obtained by incubating the Ca-HCR imaging circuit with HeLa cells at $2 \mathrm{~h}$, which meant an inadequate Ca-HCR amplification due to an inefficient uptake of these sensing probes. With prolonged reaction time, the FRET intensity increased gradually and reached a plateau at $5 \mathrm{~h}$, and there was no tremendous FRET change after $5 \mathrm{~h}$, indicating that the Ca-HCR sensor reached to an equilibrium for intracellular ATP assay in HeLa cells. Thus $5 \mathrm{~h}$ is chosen as the optimal incubation time for Ca-HCR-amplified intracellular ATP imaging application.

(A)
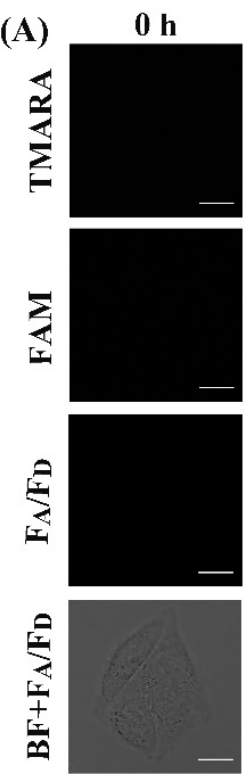

$1 \mathbf{h}$
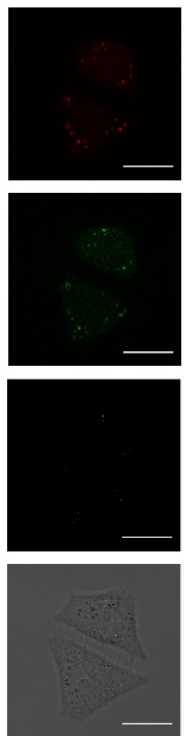

$2 \mathbf{h}$
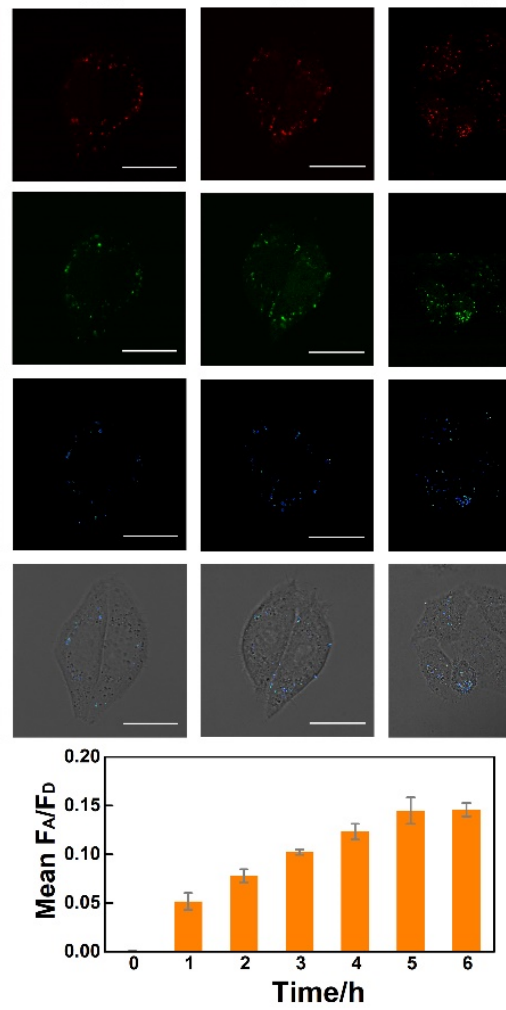

4h
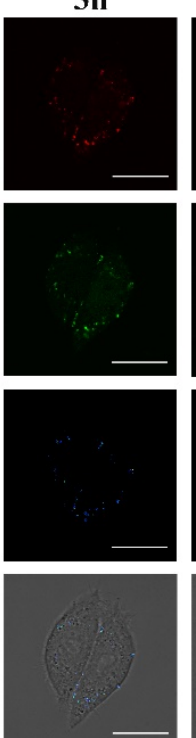
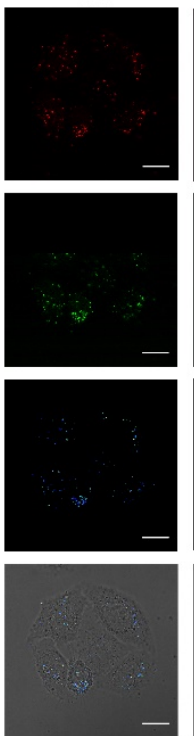

(B)
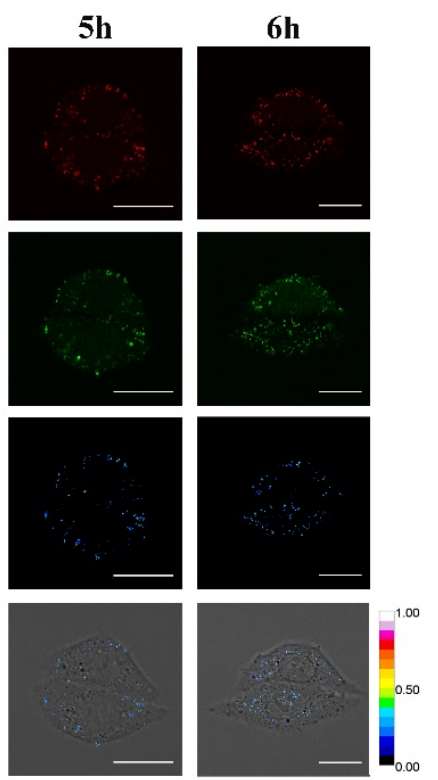

Figure S7. (A) Optimization of incubation time for the ATP-targeting Ca-HCR aptasensor in HeLa cells through intracellular imaging technique. The CLSM analysis of TMARA channel (a), FAM channel (b), FRET readout $\left(\mathrm{F}_{\mathrm{A}} / \mathrm{F}_{\mathrm{D}}\right)(\mathrm{c})$ and the merge FRET readout with bright field (d) was acquired by incubating the Ca-HCR mixture with HeLa cells for different time-intervals $(1 \mathrm{~h} \sim 6 \mathrm{~h})$ at $37^{\circ} \mathrm{C}$. (B) The corresponding fluorescence intensity $\left(\mathrm{F}_{\mathrm{A}} / \mathrm{F}_{\mathrm{D}}\right)$ of the Ca-HCR sensor was probed with different reaction durations. All scale bars corresponded to $20 \mu \mathrm{m}$. 


\section{Supporting Information}

\section{Demonstration of Ca-HCR-mediated intracellular ATP imaging}

To verify the robust amplification capacity of the Ca-HCR-amplified intracellular ATP imaging, the non-labeled hairpin $\mathbf{H}_{1}, \mathbf{H}_{2}, \mathbf{H}_{4}$ or $\mathbf{H}_{\mathbf{6}}$ of $\mathrm{Ca}-\mathrm{HCR}$ was removed for demonstrating the Ca-HCRmotivated ATP imaging (Figure S8). A significant FRET signal $\left(\mathrm{F}_{\mathrm{A}} / \mathrm{F}_{\mathrm{D}}\right)$ was obtained for the intact $\mathrm{Ca}-\mathrm{HCR}$ sensor (sample a) and was much stronger than conventional HCR imaging system $\left(\mathbf{H}_{\mathbf{6}^{-}}\right.$ excluded Ca-HCR, sample e). Moreover, scarcely any FRET readout $\left(\mathrm{F}_{\mathrm{A}} / \mathrm{F}_{\mathrm{D}}\right)$ was observed for $\mathbf{H}_{\mathbf{1}^{-}}$, $\mathbf{H}_{2}$ - or $\mathbf{H}_{4}$-excluded Ca-HCR system (sample b, c or d, respectively), indicating that all hairpin reactants were indispensable for the present Ca-HCR-mediated ATP aptasensing system. This observation not only manifested the efficient signal gain of the two-layered Ca-HCR system in living cells, but also demonstrated that the activation of $\mathrm{F}_{\mathrm{A}} / \mathrm{F}_{\mathrm{D}}$ signal was specific to the ATP aptamer substrate for activating the autonomous $\mathrm{Ca}-\mathrm{HCR}$ amplifier.

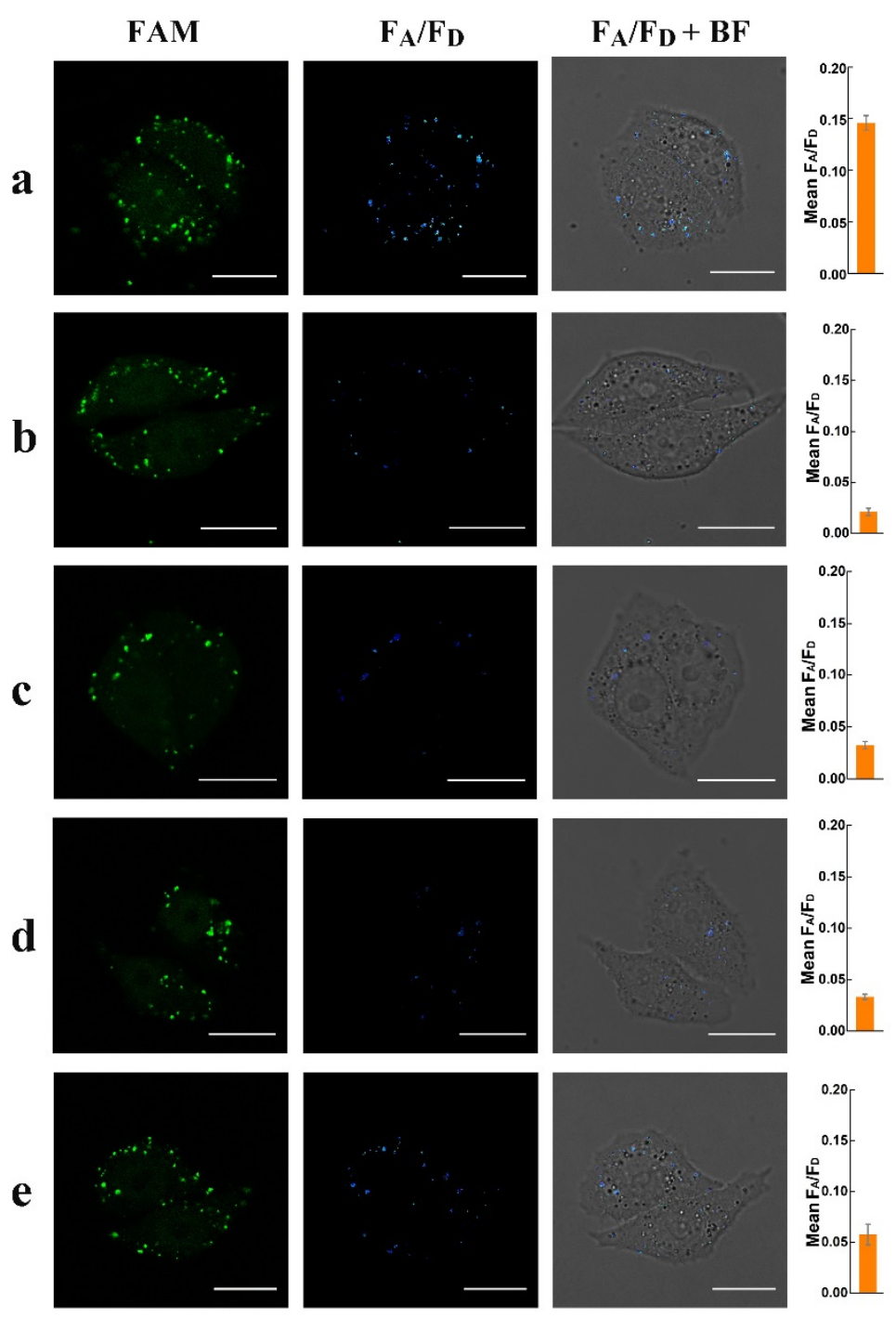

Figure S8. The images for detection of ATP in living HeLa cells by confocal laser scanning microscopy, and HeLa cells were incubated with (a) the complete Ca-HCR sensor, (b) $\mathbf{H}_{\mathbf{1}^{-}}$, (c) $\mathbf{H}_{2^{-}}$, (d) $\mathbf{H}_{4}$ - and (e) $\mathbf{H}_{6}$-excluded Ca-HCR sensor system at $37^{\circ} \mathrm{C}$ for $5 \mathrm{~h}$. Here the FRET readout signal was obtained by the ratio of FRET to FAM $\left(\mathrm{F}_{\mathrm{A}} / \mathrm{F}_{\mathrm{D}}\right)$. All the scale bars correspond to $20 \mu \mathrm{m}$. 


\section{Exploration of intracellular ATP fluctuation}

The Ca-HCR aptasensor was utilized to detect different expressions of ATP in HeLa cells. To avoid the undesired interfere from complex cellular environment, the fluorescence emission ratio of acceptor to donor $\left(\mathrm{F}_{\mathrm{A}} / \mathrm{F}_{\mathrm{D}}\right)$ was set as the FRET signal to discriminate ATP of different expression levels in HeLa cells (Figure S9). The varied ATP expression was regulated by various external stimuli. As compared with the untreated Hela cells (sample b), an obvious FRET signal was observed in HeLa cells that were treated with $10 \mathrm{mM} \mathrm{Ca}^{2+}$ (sample a), indicating that a high ATP expression in HeLa cell with $\mathrm{Ca}^{2+}$ stimulus. A comparably decreased FRET signal was observed in living HeLa cells that were treated with $300 \mathrm{nM}$ oligomycin or were incubated at $4^{\circ} \mathrm{C}$ (sample c and $\mathrm{d}$, respectively), demonstrating a low ATP expression in HeLa cells under these different conditions. These results showed that the Ca-HCR aptasensor can distinguish these different ATP expressions and fluctuations in HeLa cells under the external environment stimulation.
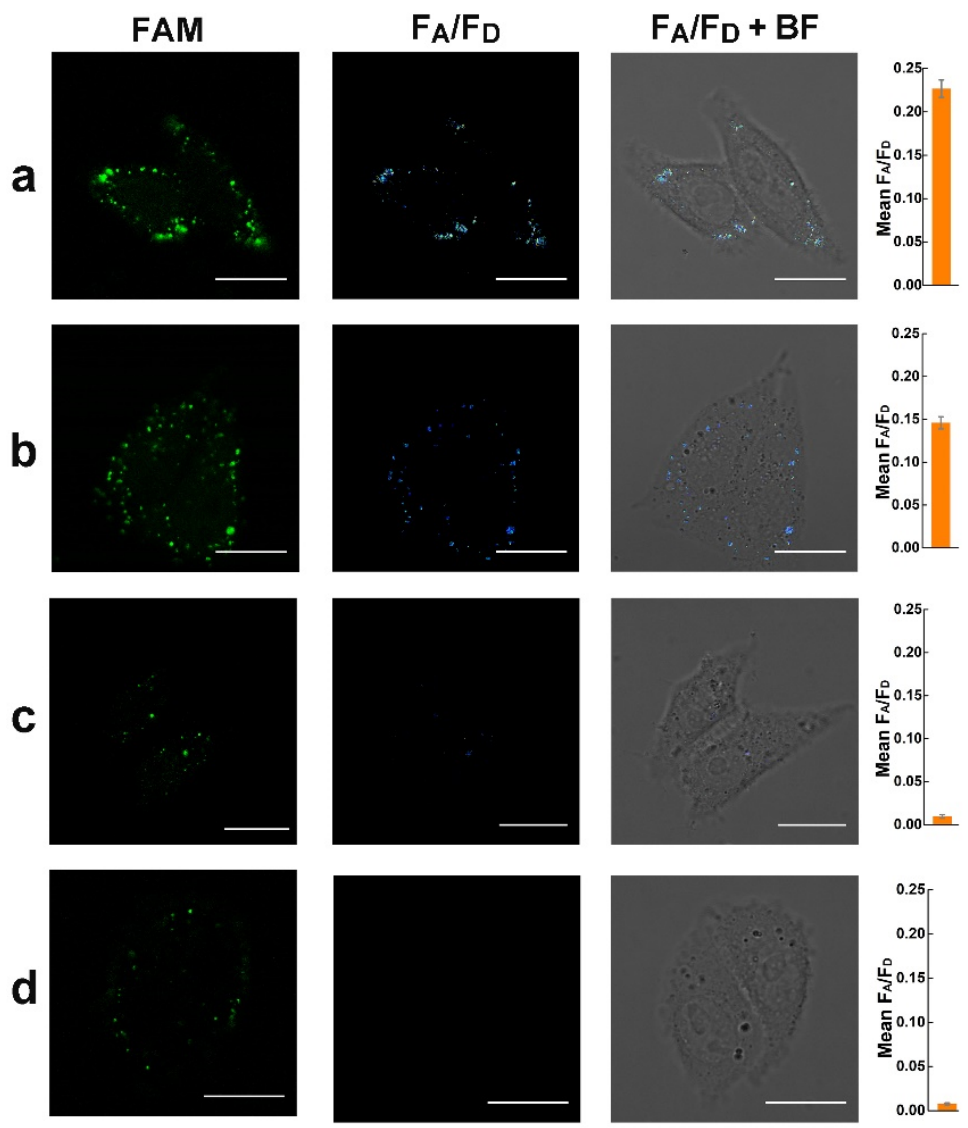

Figure S9. Living cell imaging analysis of ATP fluctuation based on our Ca-HCR-motivated FRET transduction (in the form of $F_{A} / F_{D}$ ). These CLSM images of Ca-HCR-involved ATP imaging amplifier were obtained by treating HeLa cells with $10 \mathrm{mM} \mathrm{Ca}^{2+}$ promotion (a), no treatment (b), $300 \mathrm{nM}$ oligomycin stimulation (c), or $4{ }^{\circ} \mathrm{C}$ incubation (d). The aforementioned HeLa cells were transfected and incubated with ATP-targeting Ca-HCR mixture at $37{ }^{\circ} \mathrm{C}$ for $5 \mathrm{~h}$. All scale bars corresponded to $20 \mu \mathrm{m}$. 


\section{Supporting Information}

\section{Reaction simulation of the ATP-mediated Ca-HCR sensor}

The present DNA hybridization reaction can be investigated from several derived model systems. ${ }^{1,2}$ According to Scheme 1, the ATP-initiated cascaded hybridization chain reaction can be roughly divided into several reaction processes, such as AptC-mediated ATP-recognition from AptC/AptInh hybrid to release initiator I, the upstream HCR-1 reaction to produce trigger $\mathbf{T}$, the downstream HCR-2 reaction to generate fluorescence output, the HCR-1 and HCR-2 leakages.

To simulate the ATP-aptamer recognition, the following simplifying assumptions are made:

-Assumption 1. The reaction process of the binding of ATP to the AptC strand of AptC/AptInh hybrid is irreversible for releasing the initiator $\mathbf{I}$.

-Assumption 2. The amount of the AptC/AptInh hybrid is far enough to combine ATP.

So the initiator I generation reaction can be modeled by using these following equations:

$$
\begin{gathered}
\text { AptC } / \text { AptInh }+ \text { ATP } \stackrel{K_{0}}{\rightarrow} \text { AptC } / \text { ATP }+ \text { AptInh } \\
\text { AptC } / A T P=I \\
-\frac{d[A T P]}{d t}=K_{0}[\text { AptC } / \text { AptInh }] *[\text { ATP }]
\end{gathered}
$$

For ATP, $\mathrm{C}_{(\text {AptC/AptInh) }}>>\mathrm{C}_{(\mathrm{ATP})}$, then $\mathrm{a}=[\mathrm{AptC} / \mathrm{AptInh}]=80 \mathrm{nM}$.

From equation (1), we can get:

$$
[A T P]_{t}=[A T P]_{0} * e^{-k 0 a t}
$$

To simulate the successive HCR-1/HCR-2 reaction, the following assumptions are made:

- Assumption 3. The two reactions are irreversible. This approximation is proved through multistranded partition function analysis with NUPACK (www.nupack.org).

-Assumption 4. The rate constants of all stepwise reaction are the same. This assumption is rational because all the strand-displacement reactions are mediated by 6 th toehold. The reaction rate of Ca-HCR system is mainly dominated by the length of toehold.

Based on the cross-initiation feature of HCR-1 and HCR-2 processes, the corresponding dynamic reaction can be simplified as a living polymerization procedure. Here $M_{t}$ and $N_{t}$ corresponded to the hairpin monomers' concentration over time for HCR-1 and HCR-2 reactions, respectively. I corresponds to the ATP-released initiator from AptC/AptInh hybrid. The reaction rate constants of different reaction processes are defined, including ATP recognition with its aptamer sequence, undesired self-hybridization within hairpin monomers.

The upstream HCR-1-mediated trigger $\mathbf{T}$ generation is modeled as the follows:

$$
\begin{gathered}
I^{*}+M \stackrel{K_{1}}{\longrightarrow} I \cdot M^{*} \\
I \cdot M^{*}+M \stackrel{K_{1}}{\longrightarrow} I \cdot M_{2}^{*} \\
\vdots \\
I \cdot M_{n-1}+M \stackrel{K_{1}}{\longrightarrow} I \cdot M_{n} \\
-2 * \frac{d M_{A t}}{d t}=-2 * \frac{d M_{B t}}{d t}=K_{1} *\left[I_{t}\right] *\left[M_{t}\right]
\end{gathered}
$$

The HCR-1 leakage reaction (self-hybridization of $\mathbf{H}_{\mathbf{1}}$ and $\mathbf{H}_{2}$ ) can be modeled as the follows: 


\section{Supporting Information}

$$
\begin{gathered}
M^{*}+M \stackrel{K_{M}}{\longrightarrow} M \cdot M^{*} \\
M \cdot M^{*}+M \stackrel{K_{M}}{\longrightarrow} M \cdot M_{2}^{*} \\
\vdots \\
M \cdot M_{n-1}+M \stackrel{K_{M}}{\longrightarrow} M \cdot M_{n} \\
-4 * \frac{d M_{A t}}{d t}=-4 * \frac{d M_{B t}}{d t}=K_{M} *\left[M_{t}\right]^{2}
\end{gathered}
$$

The downstream HCR-2-mediated fluorescence readout can be modeled as follows:

$$
\begin{gathered}
T^{*}+N \stackrel{K_{2}}{\longrightarrow} T \cdot N^{*} \\
T \cdot N^{*}+N \stackrel{K_{2}}{\longrightarrow} T \cdot N_{2}^{*} \\
T \cdot N_{2}^{*}+N \stackrel{K_{2}}{\longrightarrow} T \cdot N_{3}^{*} \\
T \cdot N_{3}^{*}+N \stackrel{K_{2}}{\longrightarrow} T \cdot N_{4}^{*} \\
\vdots \\
T \cdot N_{n-1}+N \stackrel{K_{2}}{\longrightarrow} T \cdot N_{n} \\
-4 * \frac{d N_{A t}}{d t}=-4 * \frac{d N_{B t}}{d t}=-4 * \frac{d N_{C t}}{d t}=-4 * \frac{d N_{D t}}{d t}=K_{2} *\left[T_{t}\right] *\left[N_{t}\right]
\end{gathered}
$$

The HCR-2 leakage reaction (self-hybridization) is modeled as follows:

$$
\begin{gathered}
N^{*}+N \stackrel{K_{N}}{\longrightarrow} N \cdot N^{*} \\
N \cdot N^{*}+N \stackrel{K_{N}}{\longrightarrow} N \cdot N_{2}^{*} \\
N \cdot N_{2}^{*}+N \stackrel{K_{N}}{\longrightarrow} N \cdot N_{3}^{*} \\
N \cdot N_{3}^{*}+N \stackrel{K_{N}}{\longrightarrow} N \cdot N_{4}^{*} \\
\vdots \\
N \cdot N_{n-1}+N \stackrel{K_{N}}{\longrightarrow} N \cdot N_{n} \\
-16 * \frac{d N_{C t}}{d t}=-16 * \frac{d N_{D t}}{d t}=-16 * \frac{d N_{E t}}{d t}=-16 * \frac{d N_{F t}}{d t}=K_{N} *\left[N_{t}\right]^{2}
\end{gathered}
$$

These equations represent the upstream HCR-1 and downstream HCR-2 reactions. To trace the time-dependent concentration changes of monomer $[\mathbf{M}]$ in lead-in HCR-1 reaction, the equations (3) and (4) are made up of two parallel processes: the routine ATP-released initiator I for HCR-1 reaction $K_{1} *\left[\mathrm{I}_{\mathrm{t}}\right] * \mathrm{M}_{\mathrm{t}}$, and the undesired self-hybridization reaction $\mathrm{K}_{\mathrm{M}} *\left[\mathrm{M}_{\mathrm{t}}\right]^{2}$. Similarly, to trace the time-dependent concentration changes of monomer [N] in downstream HCR-2 reaction, the equation (5) and (6) are composed of two parallel parts: routine HCR-1-triggered HCR-2 reaction $\mathrm{K}_{2} *\left[\mathrm{~T}_{\mathrm{t}}\right]^{*}\left[\mathrm{~N}_{\mathrm{t}}\right]$, and the undesired self-hybridization reaction $\mathrm{K}_{\mathrm{N}} *\left[\mathrm{~N}_{\mathrm{t}}\right]^{2}$. Therefore, the successive HCR-1 and HCR-2 reaction can be integrated as follows:

$$
\begin{aligned}
-\frac{d M_{t}}{d t} & =K_{1} *\left[I_{t}\right] *\left[M_{t}\right]+K_{M} *\left[M_{t}\right]^{2} \\
-\frac{d N_{t}}{d t} & =K_{2} *\left[T_{t}\right] *\left[N_{t}\right]+K_{N} *\left[N_{t}\right]^{2}
\end{aligned}
$$

Note that $\mathrm{T}_{\mathrm{t}}$ correspond to the concentration of HCR-1-generated trigger $\mathbf{T}$. The rate constants $\mathbf{K}_{\mathbf{1}}, \mathbf{K}_{\mathbf{2}}, \mathbf{K}_{\mathbf{M}}$ and $\mathbf{K}_{\mathbf{N}}$ correspond to the processes of reconstructed initiator I, HCR-2-generated-T, 


\section{Supporting Information}

HCR-1 signal leakage, and HCR-2 signal leakage, respectively. $\mathrm{M}_{0}$ and $\mathrm{N}_{0}$ represent the initial concentrations of HCR-1 and HCR-2 monomers. The ATP-consumed and M-consumed monomers are converted to $\mathbf{I}$ and $\mathbf{T}$ strands, respectively. Then the $\mathrm{I}_{\mathrm{t}}$ and $\mathrm{T}_{\mathrm{t}}$ concentrations can be given by:

$$
\begin{gathered}
{\left[I_{t}\right]=\left[A T P_{\text {consumed }}\right]=\left[A T P_{0}\right]-\left[A T P_{t}\right]} \\
{\left[T_{t}\right]=\left[M_{\text {consumed }}\right]=\left[M_{0}\right]-\left[M_{t}\right]}
\end{gathered}
$$

Thus the equations (7) and (8) can be represented as:

$$
\begin{gathered}
-\frac{d M_{t}}{d t}=\frac{1}{2} K_{1} *\left[A T P_{0}\right] *\left[M_{t}\right]-\frac{1}{2} K_{1} *\left[A T P_{0}\right] *\left[M_{t}\right] * e^{-K_{0} a t}+\frac{1}{4} K_{M} *\left[M_{t}\right]^{2} \\
-\frac{d N_{t}}{d t}=\frac{1}{4} K_{2} *\left[M_{0}\right] *\left[N_{t}\right]-\frac{1}{4} K_{2} *\left[M_{t}\right] *\left[N_{t}\right]+\frac{1}{16} K_{N} *\left[N_{t}\right]^{2}
\end{gathered}
$$

The kinetics of cascade HCR is followed with the fluorophore-labeled $\mathbf{H}_{3}$ and $\mathbf{H}_{5}$ via FRET mechanism, and the time-dependent fluorescence changes could be correlated with HCR-2 monomer as shown in the following equations:

$$
\begin{gathered}
\frac{d F_{t}}{d t}=\alpha * \frac{d N_{t}}{d t} \\
F_{t}=\propto N_{\text {consumed }}=\propto\left(N_{0}-N_{t}\right)+F_{0}
\end{gathered}
$$

Where $\alpha$ is a coefficient constant between fluorescence intensity and HCR-2 monomer's concentration. The hairpin monomer $\left([\mathrm{N}]_{0}=200 \mathrm{nM}\right)$ is completely consumed in a final equilibrium state $\left([\mathrm{N}]_{\infty}=0 \mathrm{nM}\right)$. The coefficient constant $(\alpha)$ was acquired from the fluorescence readout as follows:

$$
\alpha=\left(F_{\infty}-F_{0}\right) /\left(2 \times 10^{-7}\right)
$$

Hence, equations (11), (12) and (13) represent the ATP-motivated cascaded HCR reaction as revealed by FRET mechanism. The differential equations were simulated by using the ODE45 solver of the software MATLAB R2014a (MathWorks, Natick, MA). The experimental results showed a nonlinear fitting relationships between the time-dependent fluorescence changes and the concentration of ATP. As expected, these experimental results (open circles) showed an excellent agreement with the simulated curves (solid lines) as shown in Figure 3D.

Table S1 summarizes the average rate constants of the reaction model as acquired from the theoretical simulations of the Ca-HCR system. Interestingly, the order magnitude of the calculated parameters is consistent with the literature, ${ }^{3,4}$ implying the reliability of our reaction model. 
Table S1. Reaction constants as acquired from the theoretical simulations

\begin{tabular}{cccccc}
\hline Parameter & $\mathbf{K}_{\mathbf{0}}$ & $\mathbf{K}_{\mathbf{1}}$ & $\mathbf{K}_{\mathbf{2}}$ & $\mathbf{K}_{\mathbf{M}}$ & $\mathbf{K}_{\mathbf{N}}$ \\
\hline $\begin{array}{c}\text { Value } \\
\left(\mathrm{M}^{-1} \mathrm{~min}^{-1}\right)\end{array}$ & 25 & 623000 & 452000 & 100 & 101 \\
\hline
\end{tabular}




\section{Supporting Information}

Table S2. DNA sequences of the Ca-HCR-amplified ATP aptasensor

\begin{tabular}{|c|c|}
\hline No. & Sequence $\left(5^{\prime} \rightarrow 3^{\prime}\right)$ \\
\hline AptC & TCTACCTACGTAGCCCTGAATTCCACCTGGGGGAGTATTGCGGAGGAAGGT \\
\hline AptInh & CAGGTGGAATTCAG \\
\hline $\mathbf{H}_{1}$ & GGAATTCAGGGCTACGTAGGTAGAGTAATGCCGTCTACCTACGTAGCCCTG \\
\hline $\mathbf{H}_{2}$ & $\begin{array}{l}\text { GCTTCATCTTCATCTCCGTCTACCTACGTAGCCCTGAATTCCCAGGGCTACGTA } \\
\text { GGTAGACGGCATTACACACTC }\end{array}$ \\
\hline $\mathbf{H}_{3}$ & $\begin{array}{l}\text { GAGTGTCGGAGATGAAGATGAAGCCATCGTGCTTCATCTTCATCTCCG- } \\
\text { TAMRA }\end{array}$ \\
\hline $\mathbf{H}_{4}$ & GCTTCATCTTCATCTCCGGTTTTGCGGAGATGAAGATGAAGCACGATG \\
\hline $\mathbf{H}_{5}$ & FAM-CAAAACCGGAGATGAAGATGAAGCTTGCCTGCTTCATCTTCATCTCCG \\
\hline $\mathbf{H}_{6}$ & GCTTCATCTTCATCTCCGACACTCCGGAGATGAAGATGAAGCAGGCAA \\
\hline $\mathbf{T}$ & GCTTCATCTTCATCTCCGACACTC \\
\hline
\end{tabular}


Table S3. Summary of the different fluorescent ATP aptasensors

\begin{tabular}{cccc}
\hline Detection strategy & Detection limit & Cell imaging & Ref. \\
\hline $\begin{array}{c}\text { Exo-III-assisted target recycling } \\
\text { GO-loading DNAzyme assisted strand } \\
\text { recycling }\end{array}$ & $250 \mathrm{nM}$ & $\sqrt{ }$ & 5 \\
$\begin{array}{c}\text { RhB/ZIF-90-based nanoprobe } \\
\text { Ratiometric DNA nanoswitch }\end{array}$ & $25 \mu \mathrm{nM}$ & $\sqrt{ }$ & 7 \\
$\begin{array}{c}\text { HCR-assembled DNA hydrogel } \\
\text { Cascaded HCR amplification }\end{array}$ & $1 \mathrm{mM}$ & $\sqrt{ }$ & 8 \\
\hline
\end{tabular}




\section{Supporting Information}

\section{References}

(1) Gao, Z.; Xia, H.; Johnson, A. Nano Lett. 2018, 18, 3509-3515.

(2) Ma, P.; Liang, C.; Zhang, H.; Yin, B.; Ye, B. Chem. Sci. 2018, 9, 3299-3304.

(3) Zou, L.; Wu, Q.; Zhou, Y.; Gong, X.; Liu, X.; Wang, F. Chem. Commun. 2019, 55, 6519-6522.

(4) Biniuri, Y.; Albada, B.; Willner, I. J. Phys. Chem. B. 2018, 122, 9102-9109.

(5) Liu, X.; Freeman, R.; Willner; I. Chem. Eur. J. 2012, 18, 2207-2211.

(6) Gao, F.; Wu, J.; Yao, Y.; Zhang, Y.; Liao, X.; Genga, D.; Tang, D. RSC Adv. 2018, 8, 2816128171 .

(7) Deng, J.; Wang, K.; Wang, M.; Yu, P.; Mao, L. J. Am. Chem. Soc. 2017, 139, 5877-5882.

(8) Yuan, Jing.; Deng, Z.; Liu, H.; Li, X.; Li, J.; He, Yao.; Qing, Z.; Yang, Y.; Zhong, S. ACS Sens. 2019, 4, 1648-1653.

(9) Liao, W., Lilienthal, S.; Kahn, J. S.; Riutin, M.; Sohn, Y. S.; Nechushtai, R.; Willner, I. Chem.Sci. 2017, 8, 3362-3373. 